\title{
Eosinophilic Granulomatosis with Polyangiitis of the Major Salivary Glands: A Case of Sialadenitis in a Young Patient
}

\author{
Francesco Tovoli ${ }^{1}$, Antonio Vannini ${ }^{2}$, Chiara Masi ${ }^{1}$, Tiziana Balbi ${ }^{3}$, \\ Luigi Bolondi ${ }^{1}$ and Mario Cavazza ${ }^{2}$
}

\begin{abstract}
A 23-year-old man was referred to our Emergency Department due to an acute-onset hemoptysis and the associated bilateral swelling of all of his major salivary glands. The elevated levels of systemic inflammatory markers with hypereosinophilia and the concurrent presence of multiple lung infiltrates made it difficult to perform a differential diagnosis, as these symptoms can also be indicative of infectious, autoimmune or hematologic disorders. A histological examination of the patient's left submandibular gland revealed strong clues as to the final diagnosis of eosinophilic granulomatosis with polyangiitis with an atypical clinical presentation, thus allowing for the administration of early and successful conservative therapy. The outcome of our case suggests that systemic vasculitis represents a rare but possible cause of acute bilateral sialadenitis in young patients.
\end{abstract}

Key words: eosinophilic granulomatosis with polyangiitis, Mikulicz' disease, anti-neutrophilic cytoplasmic antibodies, acute sialadenitis

(Intern Med 52: 2131-2134, 2013)

(DOI: 10.2169/internalmedicine.52.0674)

\section{Introduction}

Eosinophilic granulomatosis with polyangiitis (EGPA) was described for the first time in 1951 by Jacob Churg and Lotte Strauss as a peculiar form of vasculitis of the small and medium-sized vessels (1). It is characterized by the formation of eosinophilic and necrotizing granulomas in the vessel walls and the perivascular tissue (2). EGPA is generally associated with a history of asthma, allergic rhinitis and eosinophilia. This disease is composed of three phases: a prodromal phase with allergic manifestations (asthma, rhinitis), a second phase marked by peripheral eosinophilia and eosinophilic tissue infiltrates and a vasculitic phase in which the signs of necrotizing vasculitis become clinically evident (3).

The involvement of the salivary glands in EGPA is not common and has been described as a minor manifestation in the setting of a more severe systemic inflammatory syn- drome when reported in literature (4-6). We herein report the case of a young man who showed bilateral swelling in all of the major salivary glands which served as the prominent clinical manifestation, together with his respiratory symptoms, that led to the diagnosis of a potentially aggressive form of EGPA and to the prompt treatment.

\section{Case Report}

A 23-year-old man was admitted to our institution complaining of hemoptysis and submandibular gland enlargement, which was more prominent on the left side. His past medical history was noteworthy for asthma, for which he had been treated with antihistamines since his childhood. Upon physical examination, the patient had a blood pressure of 110/80 $\mathrm{mmHg}$ and a heart pulse rate of $102 \mathrm{bpm}$. He was pyretic (temperature $37.5^{\circ} \mathrm{C}$ ) and his oxygen saturation was $91 \%$ on room air.

The left submandibular gland was markedly enlarged, as

\footnotetext{
${ }^{1}$ Department of Medical and Surgical Sciences, University of Bologna, Italy, ${ }^{2}$ Department of Emergency, Bologna University Hospital Authority St. Orsola-Malpighi, Italy and ${ }^{3}$ Department of Hematology, Oncology and Laboratory Medicine, Bologna University Hospital Authority St. Orsola-Malpighi, Italy

Received for publication March 28, 2013; Accepted for publication May 2, 2013

Correspondence to Dr. Francesco Tovoli, francesco.tovoli@studio.unibo.it
} 


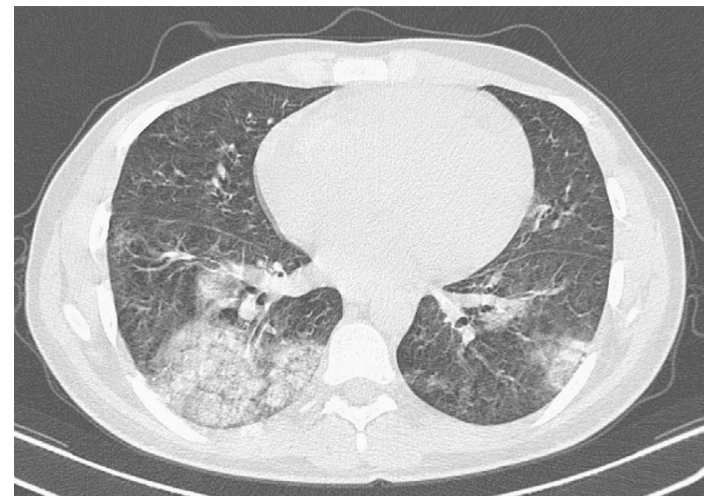

Figure 1. Chest HRCT revealed the presence of bilateral peripheral patchy areas of a ground glass consolidation.

well as tender and painful upon palpation. No enlarged cervical lymph nodes were noted. A lung auscultation revealed wheezing at the bases, but neither crackles nor rhonchi were present.

A laboratory examination revealed the following data: he-

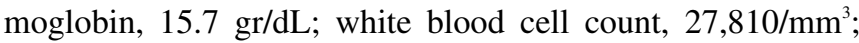
eosinophils, $16,460 / \mathrm{mm}^{3}$; erythrocyte sedimentation rate, 80 $\mathrm{mm} / \mathrm{h}$; C-reactive protein, $3.76 \mathrm{mg} / \mathrm{dL}$ (normal range: $<0.8$ $\mathrm{mg} / \mathrm{dL}$ ); IgE, $1,375 \mathrm{U} / \mathrm{mL}$ (normal range $<100 \mathrm{U} / \mathrm{mL}$ ); alanine aminotransferase, $15 \mathrm{U} / \mathrm{L}$ (normal range 8-32 U/L); aspartate aminotransferase, $20 \mathrm{U} / \mathrm{L}$ (normal range 8-37 U/L); lactate dehydrogenase, $153 \mathrm{U} / \mathrm{L}$ (normal range <240 U/L); creatine kinase, $81 \mathrm{U} / \mathrm{L}$ (normal range $<160 \mathrm{U} / \mathrm{L}$ ) and Btype natriuretic peptide, $101 \mathrm{pg} / \mathrm{mL}$ (normal range $<125 \mathrm{pg}$ / $\mathrm{mL}$ ). The kidney test results and urinalysis were normal. There was no hypergammaglobulinemia and IgG4 levels were within the normal ranges. The results for angiotensinconverting enzyme, beta2-microglobulin and salivary amylases were all normal. The assays for antinuclear antibodies (ANA), extractable nuclear antigens antibodies (ENA), antineutrophilic cytoplasmic antibodies (ANCA, tested both by indirect immunofluorescence and immunoblot) were all negative, as were the tests for antibodies to HIV, Aspergillus, Echinococcus and epidemic parotitis. No parasites were found in repeated stool tests. Chest radiography demonstrated a patchy consolidation area in the upper right lobe. Chest high-resolution computed tomography (HRCT) revealed multiple bilateral peripheral areas of ground glass consolidation (Fig. 1).

The patient underwent bronchoscopy. Lavage fluid samples demonstrated hypercellularity with eosinophilia as well as elevated levels of inflammatory cytokines (620,000 white cells/mL; 430,000 eosinophils/mL; IL-8, 1,112 pg/mL with a normal range of $<70 \mathrm{pg} / \mathrm{mL}$ ). Cultural examinations of lavage fluid were negative for bacteria, Mycobacteria and Aspergillus. No malignant cells were found.

The results of the pulmonary function tests were as follows: forced vital capacity, $5.14 \mathrm{~L}$ (90\% predicted value); forced expiratory volume in the first second, $2.63 \mathrm{~L}(55 \%$ predicted value) and Tiffenau index, 0.51. These findings were consistent with a moderate obstructive disease.
The bilateral swelling and enlargement of the submandibular glands as well as an inflammatory thickening of the paranasal sinuses were noted on the neck computed tomography images (Fig. 2). Scintigraphy revealed a significant hypofunction of the parotid, submandibular and sublingual glands. A biopsy of the left submandibular gland showed eosinophilic granulomas with dendritic cells and histiocytic macrophages.

The patient underwent left submandibular sialoadenectomy surgery. A histological examination revealed the presence of interstitial fibrosis with mixed CD3+ and CD20+ follicular lymphoplasmacellular infiltrates as well as periductal inflammatory infiltrate with a rich eosinophilic component. Evidence of a lymphoepithelial lesion was also found and subsequently confirmed with anti-CD3 staining (Fig. 3). MIB-1 immunoreactivity testing revealed a low lymphocytic growth fraction, which is consistent with a diagnosis of reactive infiltrate. No IgG4 deposits were found using the appropriate staining. A bone marrow biopsy sample was normocellular for age and lacked a clear granulopoietic prevalence, which is inconsistent with a diagnosis of hypereosinophilic syndrome. An echocardiogram was obtained and did not show any pathologic abnormalities.

The patient was treated with methylprednisolone at a dosage of $1 \mathrm{mg} / \mathrm{kg}$ intravenously for 3 days followed by a dosing strategy of $0.5 \mathrm{mg} / \mathrm{kg} /$ day. After 7 days of treatment, his eosinophil count normalized and he began to improve, both clinically and radiologically. An HRCT performed one month later showed the complete resolution of the pulmonary ground glass areas and a marked volume reduction in the residual salivary glands.

\section{Discussion}

Our patient was diagnosed to have EGPA because five out of the six American College of Rheumatology criteria for the condition were satisfied (asthma, eosinophilia $>10 \%$, paranasal sinusitis, pulmonary infiltrates and histological proof of vasculitis) (7). Although EGPA is considered to be among the ANCA-associated vasculitides, only a minority of patients (35-40\%) have detectable circulating ANCA levels, therefore, a negativity for these autoantibodies in our patient was not surprising (8). It has been reported that ANCAnegative patients have a different subset of disease, in which lung infiltrates and systemic vasculitis features are predominant, whereas ANCA-positive patients are more prone to renal or peripheral nervous system involvement (8).

Consistent with these observations, our patient's main clinical features consisted of eosinophilic vasculitis of the salivary glands and patchy consolidation areas in both of the lungs, without renal or nervous system involvement. Lung manifestations are very common and are regarded as a keystone in the diagnosis of EGPA. These manifestations can range from the presence of patchy and usually transient infiltrates to eosinophilic abscess or even the rare but severe forms of alveolar hemorrhage (9). 

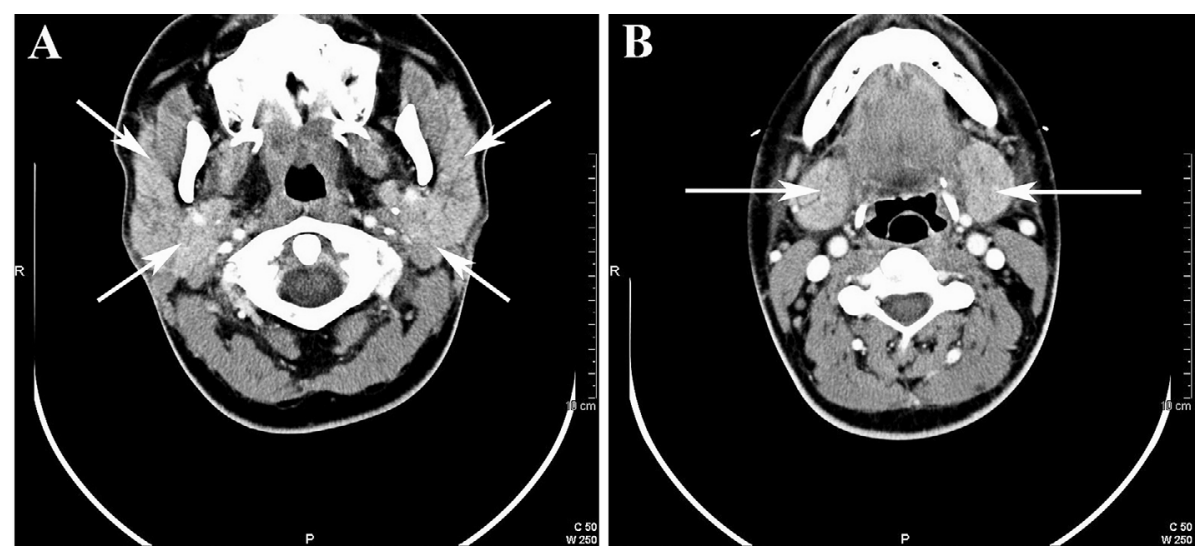

Figure 2. Swelling of the major salivary glands is evident on the neck CT. In particular, both parotid glands are moderately enlarged $(A)$ and both submandibular glands are markedly increased in volume (B).
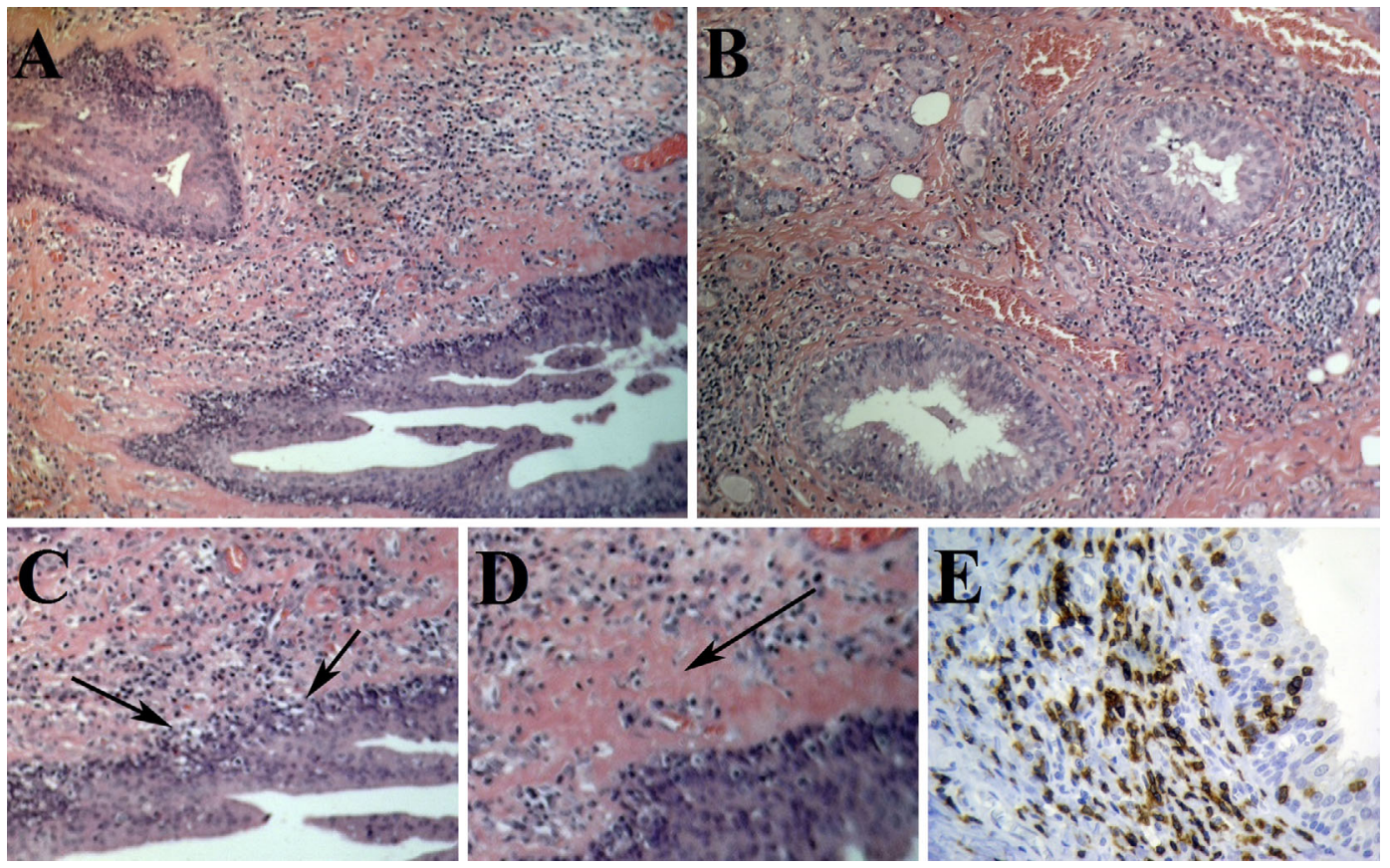

Figure 3. The histological samples obtained from the left submandibular gland $(400 \times)$, showing the perivascular/periductal eosinophilic and lymphocytic infiltrate $(A, B)$, eosinophilic infiltration of a salivary duct (C) and interstitial fibrosis with follicular lymphocytic infiltrate (D). Anti-CD3 staining confirmed the presence of a lymphoepithelial lesion ( $E)$.

On the other hand, the enlargement of the salivary glands is not a typical feature of EGPA, and has been reported only rarely (4-6). In fact, in the first cases reported by Gambari and Boin $(4,5)$, the swelling of the salivary glands was interpreted as a feature of primary vasculitic involvement. More recently, two cases of patients with EGPA as well as features of Mikulicz's disease (MD) have been reported $(6,10)$.

Although MD was long considered to be associated with primary Sjögren Syndrome, the condition was recently identified as an IgG4-related disease (IgG4-RD) and is now clinically characterized by the persistent swelling of the lacrimal and salivary glands (11). Furthermore, a recent study undertaken by Yamamoto et al. found elevated $\mathrm{IgG} 4$ serum levels in cases of MD and cases of EGPA (12), thus suggesting that there may be a possible underlying pathogenic link between these two different diseases. To our knowledge, in previously reported cases of patients with EGPA and features of MD, it has never been specified if the typical histological features of IgG4-RD were found at the microscopic examination of major salivary glands.

In our patient, the submandibular gland histology was characterized by a prevalently polinuclear infiltrate with eosinophils migrating through the blood vessels and forming eosinophilic granulomas with scanty lymphoplasmacytic infiltrate. Therefore, the histology was strongly suggestive for EGPA granulomas and not consistent with a diagnosis of IgG4-RD. A coexistent IgG4-RD was also ruled out by the 
presence of normal IgG4 serum levels.

Similar findings have been reported for a differential diagnosis of Kimura disease, a medical condition that is characterized by the presence of subcutaneous nodules in the head and neck with the involvement of the cervical lymph nodes and salivary glands. These patients also have eosinophilia in their peripheral blood as well as tissue eosinophil inflammatory infiltrates (13). Furthermore, lung eosinophilic infiltrates are a rare but possible condition in this setting (14). However, in our case, the histological examination of the submandibular gland did not show the other prominent features of Kimura disease. In particular, there were no signs of florid reactive follicles, marked germinal vascularization, eosinophilic abscesses or vascular hyalinization with proteinaceous deposits. In addition, the polyclonality of the submandibular gland lymphoplasmacytic infiltrate and its low growth fraction ruled out the more severe forms of lymphoproliferative disorders that may be associated with peripheral eosinophilia (i.e. Hodgkin's lymphoma).

The present case deals with the uncommon presentation of a rare and potentially aggressive disease. Salivary gland enlargement, although unusual, can be the initial clinical presentation of EGPA, which sometimes mimics other, more common, causes of acute sialadenitis such as infectious sialadenitis. The inflammation of the major salivary glands in the setting of EGPA may represent a primary vasculitis involvement (as in our case), or be expressions of a concomitant IgG4-RD, such as MD. Our findings suggest that vasculitis should be considered among the differential diagnoses for patients with enlargements of the salivary glands in addition to other systemic symptoms. In this setting, the salivary glands may also offer an easy route for a confirmatory biopsy. In our case, the presence of eosinophilic granulomas in a submandibular gland were a strong clue to the underlying diagnosis, thus leading the way to administer timely therapy and to thereby obtain a successful outcome.

\section{The authors state that they have no Conflict of Interest (COI).}

\section{References}

1. Churg J, Strauss L. Allergic granulomatosis, allergic angiitis and polyarteritis nodosa. Am J Pathol 27: 277-301, 1951.

2. Jennette JC, Falk RJ, Andrassy K, et al. Nomenclature of systemic vasculitides: proposal of an international consensus conference. Arthritis Rheum 37: 187-192, 1994.

3. Lanhssm JG, Elkon KB, Pusey CD, Hughes GR. Systemic vasculitis with astma and eosinophilia: a clinical approach to the ChurgStrauss syndrome. Medicine (Baltimore) 63: 65-81, 1984.

4. Gambari PF, Ostuni PA, Lazzarin P, Fassina A, Todesco S. Eosinophilic granuloma and necrotizing vasculitis (Churg-Strauss syndrome?) involving a parotid gland, lymph nodes, liver and spleen. Scand J Rheumatol 18: 171-175, 1989.

5. Boin F, Sciubba JJ, Stone JH. Churg-Strauss syndrome presenting with salivary gland enlargement and respiratory distress. Arthritis Rheum 55: 167-170, 2006.

6. Ayuzawa N, Ubara Y, Keiichi S, et al. Churg-Strauss syndrome with a clinical condition similar to IgG4-related kidney disease: a case report. Intern Med 51: 1233-1238, 2012.

7. Masi AT, Hunder GG, Lie JT, et al. The American College of Rheumatology 1990 criteria for the classication of Churg-Strauss syndrome (allergic granulomatosis and angiitis). Arthritis Rheum 33: 1094-1100, 1990.

8. Sablé-Fourtassou R, Cohen P, Mahr A, et al. Antineutrophil cytoplasmic antibodies and the Churg-Strauss syndrome. Ann Intern Med 143: 632-638, 2005.

9. Pagnoux C. Churg-Strauss syndrome: evolving concepts. Discov Med 9: 243-252, 2010.

10. Hanioka Y, Yamagami K, Yoshioka K, et al. Churg-Strauss syndrome concomitant with chronic symmetrical dacryoadenitis suggesting Mikulicz's disease. Intern Med 51: 2457-2461, 2012.

11. Himi $T$, Takano $K$, Yamamoto $M$, et al. A novel concept of Mikulicz's disease as IgG4-related disease. Auris Nasus Larynx 39: 9-17, 2012.

12. Yamamoto M, Takahashi H, Suzuki C, et al. Analysis of serum IgG subclasses in Churg-Strauss syndrome--the meaning of elevated serum levels of IgG4. Intern Med 49: 1365-1370, 2010.

13. Iwai $H$, Nakae $K$, Ikeda $K$, et al. Kimura disease: diagnosis and prognostic factors. Otolaryngol Head Neck Surg 137: 306-311, 2007.

14. Koh H, Kamiishi N, Chiyotani A, et al. Eosinophilic lung disease complicated by Kimura's disease: a case report and literature review. Intern Med 51: 3163-3167, 2012.

(C) 2013 The Japanese Society of Internal Medicine http://www.naika.or.jp/imonline/index.html 